http://dx.doi.org/10.21611/qirt.2017.035

\title{
Fabrication and characterization of InGaAsSb/GaSb photodetectors for SWIR detection
}

\author{
Jun Oh Kim ${ }^{1 \dagger}$, Nguyen Tien Dai ${ }^{1}$, Jehwan Hwang ${ }^{1}$, Sang Jun Lee ${ }^{1^{*}}$ \\ ${ }^{1}$ Korea Research Institute of Standards and Science, Daejeon 34113, Korea \\ ${ }^{\dagger}$ Presenting Author: jokim@kriss.re.kr \\ *Corresponding Author: sjlee@kriss.re.kr
}

\begin{abstract}
We report on the short wavelength infrared (SWIR) photodetector based on quaternary InGaAsSb. The InGaAsSb samples were grown by molecular beam epitaxy (MBE) system on a GaSb substrate. The InGaAsSb layers were lattice-matched to the GaSb substrate. We took a high resolution X-ray diffractometer (HR-XRD) to investigate the composition and structural quality of InGaAsSb epilayers. In order to measure the optical properties of infrared photodetectors, the InGaAsSb devices were fabricated in $410 \times 410 \mu \mathrm{m}^{2}$ using conventional photolithography. We have measured the spectral response of InGaAsSb based photodetector at room temperature. The cut-off wavelength of photodetector with different composition of InGaAsSb absorption layer was $2.5 \mu \mathrm{m}$ and $3.0 \mu \mathrm{m}$ at room temperature.
\end{abstract}

KEYWORDS: Short wavelength infrared, Molecular beam epitaxy, photodetector, InGaAsSb

\section{INTRODUCTION}

Short wavelength (1-3 $\mu \mathrm{m})$ infrared (SWIR) detectors have been received great attention for a variety of applications in military and industrial such as remote sensing, molecular spectroscopy, environmental monitoring, gas detection and night vision.[1] Currently, SWIR detection systems are predominantly based on type-II InAs/GaSb superlattice (T2SL), mercury cadmium telluride (MCT) and extended indium gallium arsenide (EX-InGaAs) technologies. However, MCT have intrinsic draw-back such as inferior material uniformity.[2] For Ex-InGaAs, the material quality is degraded due to increase misfit dislocation density introduced by the lattice-mismatch. Over the past years, the quaternary InGaAsSb materials have been researched for SWIR detection.[3] Recent progress in InGaAsSb technology has made it a promising candidate for high-performance infrared detection in the SWIR due to high speed and lower noise.

\section{EXPERIMENTAL}

In this work, we have grown, processed, and characterized two InGaAsSb photodetectors grown by molecular beam epitaxy (MBE). The structural quality of InGaAsSb epi layer was performed using HR-XRD. The spectral response was measured using a glow-bar source within the Nicholet 570 Fourier transform infrared spectrometer (FTIR).

\subsection{MBE GROWTH OF PHOTODETECTORS}

The samples of InGaAsSb infrared photodetector were grown on n-type GaSb substrate using a molecular beam epitaxy with an $\mathrm{As}_{2}$ and $\mathrm{Sb}_{2}$ valve cracker source. Fig. 1 illustrates the layer structure of the $\mathrm{AlGaSb} / \mathrm{InGaAsSb}$ infrared photodetector. The device consisted of an n-type $\left(2 \times 10^{18} \mathrm{~cm}^{-3}\right) 300 \mathrm{~nm}$ thick GaSb buffer layer, an n-type $\left(2 \times 10^{18} \mathrm{~cm}^{-3}\right) 300 \mathrm{~nm}$ thick InGaAsSb bottom contact layer, an n-type $\left(2 \times 10^{16} \mathrm{~cm}^{-3}\right) 2000 \mathrm{~nm}$ thick InGaAsSb absorption layer, a $60 \mathrm{~nm}$ unintentionally doped $\mathrm{Al}_{0.3} \mathrm{Ga}_{0.7} \mathrm{Sb}$ layer and an n-type $\left(2 \times 10^{18} \mathrm{~cm}^{-3}\right) 200 \mathrm{~nm}$ thick InGaAsSb for top contact layer. The $\mathrm{Al}_{0.3} \mathrm{Ga}_{0.7} \mathrm{Sb}_{\text {barrier was used to }}$ create an $\mathrm{nBn}$ detector, which has a large conduction band offset compare to the InGaAsSb absorber. Two InGaAsSb samples were grown on GaSb substrate, where the composition of In and As were 28\%, 25\% (sample A) and 17\%, 14\% (sample B), respectively. After growth, the samples were characterized using HR-XRD. Diffraction patterns were obtained from the (004) reflection. The results indicated that the $\mathrm{In}_{0.28} \mathrm{Ga}_{0.72} \mathrm{As}_{0.25} \mathrm{Sb}_{0.75}$ layer was lattice matched to the GaSb substrate as shown in the Fig. 1, where the $\operatorname{In}_{0.17} \mathrm{Ga}_{0.83} \mathrm{As}_{0.14} \mathrm{Sb}_{0.86}$ sample was lattice mismatched to substrate. 

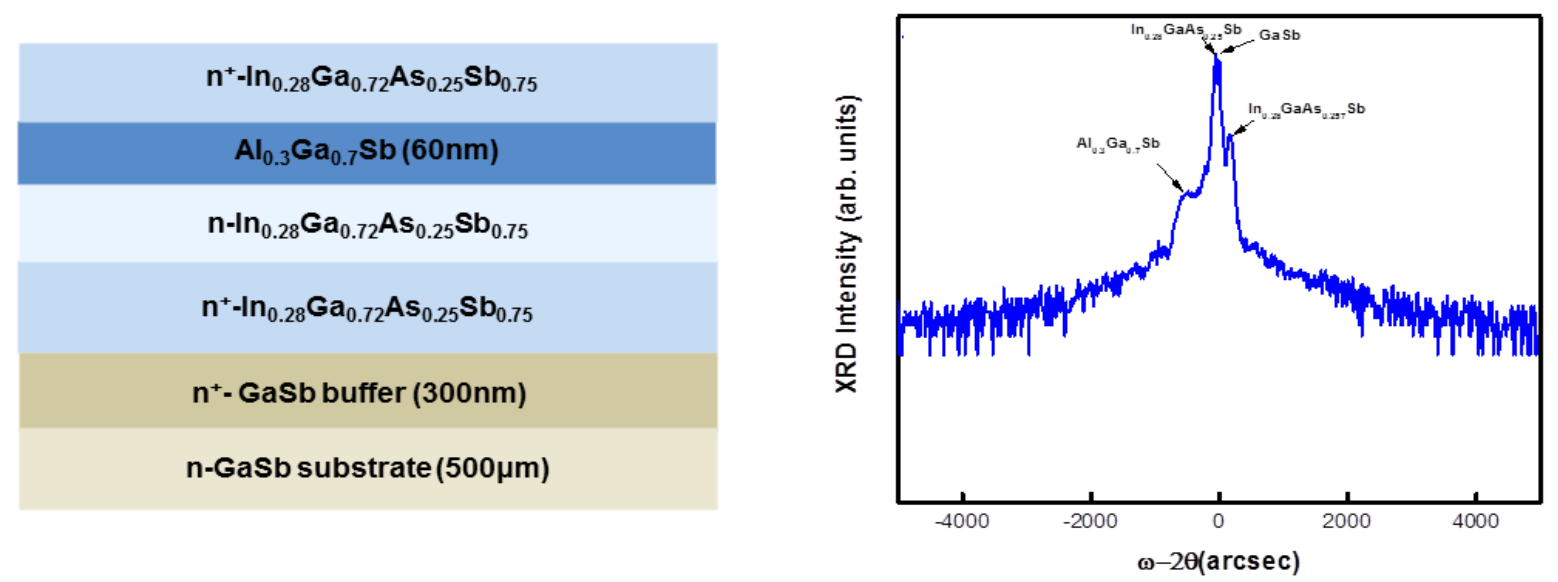

Fig. 1 Schematic view of InGaAsSb photodetector and HR-XRD result

\subsection{DEVICE FABRICATION AND CHARACTERIZATION}

The InGaAsSb devices were performed in $410 \times 410 \mu \mathrm{m}^{2}$ using a photolithography for the front-illuminated structure, where the device had a circular aperture of $300 \mu \mathrm{m}$ in each mesa. Afterwards, the ohmic contact metals of $\mathrm{Ge} / \mathrm{Au} / \mathrm{Ni} / \mathrm{Au}$ were deposited on the top and bottom contact layer. Subsequently, the contact annealing at $380{ }^{\circ} \mathrm{C}$ was performed on the devices using rapid thermal annealing. The Spectral response measurement was performed using a glow-bar source within the Nicholet 570 Fourier transform infrared spectrometer (FTIR) as a function of temperature. The photodetectors were measured under zero voltage bias. Two samples were characterized in order to compare the device performances. The cut-off wavelength of sample A and B was $3.0 \mu \mathrm{m}$ and $2.5 \mu \mathrm{m}$ at room temperature, which is due to the bandgap of absorber material.

\section{CONCLUSIONS}

In conclusion, the two nBn photodetectors, one with lattice-matched (sample A) and the other lattice-mismatched (sample B) to the substrate, were demonstrated based on quaternary InGaAsSb material and AlGaSb barrier layer. The structures were grown using the molecular beam epitaxy technology. The HR-XRD indicated good material quality of the InGaAsSb layer. Room temperature spectral response of sample A and B were obtained with the cutoff wavelength at $3.0 \mu \mathrm{m}$ and $2.5 \mu \mathrm{m}$, respectively.

\section{ACKNOWLEDGMENT}

This work was supported by the KRISS grant GP2017-0031 and the AOARD grant FA2386-14-1-4094 funded by the U.S. government (AFOSR/AOARD).

\section{REFERENCES}

[1] B. Carter, E. Shaw, J. Olesberg, W. Chan, T. Hasenberg, M. Flatte, High detectivity InGaAsSb pin infrared photodetector for blood glucose sensing, Electronics Letters, 36 (2000) 1301-1303.

[2] J.D. Phillips, K. Moazzami, J. Kim, D.D. Edwall, D.L. Lee, J.M. Arias, Uniformity of optical absorption in HgCdTe epilayer measured by infrared spectromicroscopy, Applied Physics Letters, 83 (2003) 3701.

[3] H. Shao, A. Torfi, W. Li, D. Moscicka, W.I. Wang, High detectivity AlGaAsSb/InGaAsSb photodetectors grown by molecular beam epitaxy with cutoff wavelength up to 2.6, Journal of Crystal Growh, 311 (2009) 1893-1896. 